\title{
Hybrid Bernstein Block-Pulse Functions Method for Second Kind Integral Equations with Convergence Analysis
}

\author{
Mohsen Alipour, ${ }^{1}$ Dumitru Baleanu, ${ }^{2,3,4}$ and Fereshteh Babaei ${ }^{1}$ \\ ${ }^{1}$ Faculty of Basic Science, Babol University of Technology, P.O. Box 47148-71167, Babol, Iran \\ ${ }^{2}$ Department of Mathematics, Cankaya University, Ogretmenler Caddesi 14, Balgat, 06530 Ankara, Turkey \\ ${ }^{3}$ Institute of Space Sciences, P.O. Box MG 23, Magurele, 077125 Bucharest, Romania \\ ${ }^{4}$ Department of Chemical and Materials Engineering, Faculty of Engineering, King Abdulaziz University, \\ P.O. Box 80204, Jeddah 21589, Saudi Arabia
}

Correspondence should be addressed to Mohsen Alipour; m.alipour2323@gmail.com

Received 22 November 2013; Accepted 9 January 2014; Published 23 February 2014

Academic Editor: Carlo Cattani

Copyright (C) 2014 Mohsen Alipour et al. This is an open access article distributed under the Creative Commons Attribution License, which permits unrestricted use, distribution, and reproduction in any medium, provided the original work is properly cited.

\begin{abstract}
We introduce a new combination of Bernstein polynomials (BPs) and Block-Pulse functions (BPFs) on the interval [0,1]. These functions are suitable for finding an approximate solution of the second kind integral equation. We call this method Hybrid Bernstein Block-Pulse Functions Method (HBBPFM). This method is very simple such that an integral equation is reduced to a system of linear equations. On the other hand, convergence analysis for this method is discussed. The method is computationally very simple and attractive so that numerical examples illustrate the efficiency and accuracy of this method.
\end{abstract}

\section{Introduction}

In recent years, many different basic functions have been used for solving integral equations, such as Block-Pulse functions $[1,2]$, Triangular functions [3], Haar functions [4], Hybrid Legendre and Block-Pulse functions [5], Hybrid Chebyshev and Block-Pulse functions [6, 7], Hybrid Taylor and BlockPulse functions [8], and Hybrid Fourier and Block-Pulse functions [9].

Block-Pulse functions were introduced in electrical engineering by Harmuth. After that study, several researchers have discussed applications of Block-Pulse functions [10, 11].

Bernstein polynomials have been applied in various fields of mathematics. For example, some researchers applied the Bernstein polynomials for solving high order differential equations [12], some classes of integral equations [13], partial differential equations, and optimal control problems [14]. Also, we introduced new operational matrices of fractional derivative and integral operators by Bernstein polynomials and then used them for solving fractional differential equations [15-17], system of fractional differential equations [18], and fractional optimal control problems $[19,20]$.

In this work, we combine the Bernstein polynomials (BPs) and Block-Pulse functions (BPFs) on the interval $[0,1]$. Then, we use these bases for finding an approximate solution of the second kind integral equation. We call this method Hybrid Bernstein Block-Pulse Functions Method (HBBPFM). In this method the integral equation is reduced to a system of linear equations. Also, we discuss the convergence analysis for this method. Furthermore, we compare the accuracy of obtained results of BPFs, BPs, and HBBPFM by some examples.

The rest of this paper is as follows. In Section 2, HBBPFs are introduced; therefore we approximate functions by using HBBPFs and also we discuss best approximation and convergence analysis in Section 3. Then we apply HBBPF method to find an approximate solution for the second kind integral equations and we survey error analysis for proposed method in Section 4. Also, we apply the proposed method on some examples. We observe that the accuracy and efficiency of this 
method are more than the near methods. Finally, Section 6 concludes our work in this paper.

\section{Hybrid of Bernstein and Block-Pulse Functions}

In this section, we recall some definitions and properties of Bernstein polynomials and Block-Pulse functions.

Lemma 1 (see [19]). The Bernstein polynomials (BPs) of mthdegree are defined on the interval $[0,1]$ as follows:

$$
B_{i, m}(x)=\left(\begin{array}{c}
m \\
i
\end{array}\right) x^{i}(1-x)^{m-i}, \quad i=0,1, \ldots, m,
$$

where

$$
\left(\begin{array}{c}
m \\
i
\end{array}\right)=\frac{m !}{i !(m-i) !}
$$

Then $\left\{B_{0, m}, B_{1, m}, \ldots, B_{m, m}\right\}$ in Hilbert space $L^{2}[0,1]$ is a complete basis. Therefore, any polynomial of degree $m$ can be expanded in terms of linear combination of $B_{i, m}(x)(i=$ $0,1, \ldots, m)$.

Lemma 2. Let a set of Block-Pulse functions (BPFs) $b_{i}(t), i=$ $1,2, \ldots, N$ be on the interval $[0,1)$ such that.

$$
b_{i}(t)= \begin{cases}1, & \frac{i-1}{N} \leq t<\frac{i}{N} \\ 0, & \text { otherwise. }\end{cases}
$$

Then, the following properties for these functions satisfy the following:
(i) disjointness,
(ii) orthogonality,
(iii) completeness.

Proof. The disjointness property can be clearly obtained from the definition of Block-Pulse functions as follows:

$$
b_{i}(t) b_{j}(t)= \begin{cases}b_{i}(t), & i=j, \\ 0, & i \neq j,\end{cases}
$$

where $i, j=1,2, \ldots, N$

The other property is orthogonality. It is clear that

$$
\int_{0}^{1} b_{i}(t) b_{j}(t) d t=\frac{1}{N} \delta_{i j}
$$

where $i, j=1,2, \ldots, N$ and $\delta_{i j}$ is the Kroneker delta.

The third property is completeness. For every $f \in$ $L^{2}([0,1))$, when $m$ approaches the infinity, Parseval's identity holds:

$$
\int_{0}^{1} f^{2}(x) d x=\sum_{i=0}^{\infty}\left(f_{i}^{2}\left\|b_{i}(t)\right\|^{2}\right)
$$

where $f_{i}=N \int_{0}^{1} f(t) b_{i}(t) d t$.
Definition 3 (Hybrid Bernstein Block-Pulse Functions (HBBPFs)). $H_{n, m}(t), n=1,2, \ldots, N, m=0,1, \ldots, M$, have three arguments; $n$ and $m$ are the order of BPFs and BPs, respectively, and $t$ is the normalized time. HBBPFs are defined on the interval $[0,1)$ as follows:

$$
H_{n, m}(t)= \begin{cases}B_{m, M}(N t-n+1), & \frac{n-1}{N} \leq t \leq \frac{n}{N}, \\ 0, & \text { otherwise. }\end{cases}
$$

In the next section, we deal with the problem of approximation of these functions.

\section{Approximation of Functions by Using HBBPFs and Convergence Analysis}

Theorem 4. Suppose that the function $f:[0,1] \rightarrow$ $R$ is $m+1$ times continuously differentiable, and $S=$ $\operatorname{Span}\left\{B_{0, m}, B_{1, m}, \ldots, B_{m, m}\right\}$. Then $c^{T} B=s_{0}=\sum_{i=0}^{m} c_{i} B_{i, m} \in S$ is the best approximation $f$ out of $S \subseteq L^{2}[0,1]$ with the following inner product:

$$
\begin{aligned}
\langle f, B\rangle & =\int_{0}^{1} f(x) B(x)^{T} d x \\
& =\left[\left\langle f, B_{0, m}\right\rangle,\left\langle f, B_{1, m}\right\rangle, \ldots,\left\langle f, B_{m, m}\right\rangle\right],
\end{aligned}
$$

where $B^{T}=\left[B_{0, m}, B_{1, m}, \ldots, B_{m, m}\right]$ and $c^{T}=\left[c_{1}, c_{2}, \ldots, c_{m}\right]$. Also, one can obtain the following inequality:

$$
\left\|f-c^{T} B\right\|_{L^{2}[0,1]} \leq \frac{\widehat{K}}{(m+1) ! \sqrt{2 m+3}},
$$

where $\widehat{K}=\max _{x \in[0,1]}\left|f^{(m+1)}(x)\right|$.

Proof. We prove that $c^{T} B$ is the best approximation for $f$ out of $S$. We can prove that $S$ is a convex subset of a real inner product space $L^{2}[0,1]$ (see [21]). Therefore, for any $x \in L^{2}[0,1], \widehat{x} \in S$ is its best approximation in $S$ if and only if it satisfies

$$
\langle x-\widehat{x}, z-\widehat{x}\rangle \leq 0 \quad \forall z \in S,
$$

where the inner product is defined by $\langle f, g\rangle=\int_{0}^{1} f(t) g(t) d t$. Then for any $x \in L^{2}[0,1]$, its best approximation is unique. Also, we know that $S \subset L^{2}[0,1]$ is a convex and closed finitedimensional subset of an inner product space $L^{2}[0,1]$. Then for any $x \in L^{2}[0,1]$, there is a unique element $\widehat{x} \in S$ such that $\|x-\widehat{x}\|=\inf _{z \in S}\|x-z\|$. Therefore, there exist the unique coefficients $c_{i}, i=0,1, \ldots, m$ such that

$$
f \cong s_{0}=\sum_{i=0}^{m} c_{i} B_{i, m}=c^{T} B .
$$

On the other hand, we can consider that $\left\{1, x, \ldots, x^{n}\right\}$ is a basis for polynomials space of degree $m$. Therefore we define $y_{1}(x)=f(0)+x f^{\prime}(0)+\left(x^{2} / 2 !\right) f^{\prime \prime}(0)+\cdots+\left(x^{m} / m !\right) f^{(m)}(0)$. Hence, from Taylor expansion we have

$$
\left|f(x)-y_{1}(x)\right|=\left|f^{(m+1)}\left(\xi_{x}\right) \frac{x^{m+1}}{(m+1) !}\right|,
$$


TABLE 1: Absolute errors by using BPFs for $N=4$, BPs for $M=3$, and HBBPFM for $N=4, M=3$ in Example 1 .

\begin{tabular}{lccc}
\hline & & Method & \\
$t$ & BPFs & BPs & HBBPFM \\
& $N=4$ & $M=3$ & $N=4, M=3$ \\
\hline 0 & 0.159448 & 0.000252739 & $2.57612 \times 10^{-7}$ \\
0.1 & 0.0596148 & 0.0000539886 & $5.73616 \times 10^{-8}$ \\
0.2 & 0.0392211 & 0.000110834 & $1.23088 \times 10^{-7}$ \\
0.3 & 0.118936 & 0.0000398714 & $3.42659 \times 10^{-7}$ \\
0.4 & 0.0250381 & 0.0000566614 & $2.06685 \times 10^{-7}$ \\
0.5 & 0.167325 & 0.000106028 & $1.3331 \times 10^{-6}$ \\
0.6 & 0.0821085 & 0.0000743689 & $3.07359 \times 10^{-7}$ \\
0.7 & 0.00253325 & 0.0000243121 & $5.58694 \times 10^{-7}$ \\
0.8 & 0.125405 & 0.000119641 & $7.24512 \times 10^{-7}$ \\
0.9 & 0.0594347 & 0.000076931 & $4.20127 \times 10^{-7}$ \\
\hline
\end{tabular}

TABLE 2: Absolute errors by using BPFs for $N=5$, BPs for $M=4$, and HBBPFs for $N=5, M=4$ in Example 1 .

\begin{tabular}{lccc}
\hline & & Method & \\
$t$ & BPFs & BPs & HBBPFM \\
& $N=5$ & $M=4$ & $N=5, M=4$ \\
\hline 0 & 0.120718 & 0.0000294061 & $9.38506 \times 10^{-9}$ \\
0.1 & 0.0208845 & 0.0000117512 & $4.43327 \times 10^{-10}$ \\
0.2 & 0.124426 & $4.28074 \times 10^{-6}$ & $7.87196 \times 10^{-9}$ \\
0.3 & 0.0275755 & $7.98903 \times 10^{-6}$ & $1.13157 \times 10^{-9}$ \\
0.4 & 0.124293 & $8.7962 \times 10^{-6}$ & $6.2511 \times 10^{-9}$ \\
0.5 & 0.034286 & $2.23581 \times 10^{-7}$ & $1.14373 \times 10^{-9}$ \\
0.6 & 0.120604 & $8.9113 \times 10^{-6}$ & $1.22382 \times 10^{-8}$ \\
0.7 & 0.0410285 & $7.57027 \times 10^{-6}$ & $1.62177 \times 10^{-9}$ \\
0.8 & 0.230475 & $7.94076 \times 10^{-6}$ & $4.74483 \times 10^{-10}$ \\
0.9 & 0.00358729 & 0.0000229948 & $9.2012 \times 10^{-9}$ \\
\hline
\end{tabular}

where $\xi_{x} \in(0,1)$. Since $c^{T} B$ is the best approximation $f$ out of $S$, and we assume that $y_{1} \in S$, therefore, we have

$$
\begin{aligned}
\left\|f-c^{T} B\right\|_{L^{2}[0,1]}^{2} & \leq\left\|f-y_{1}\right\|_{L^{2}[0,1]}^{2} \\
& =\int_{0}^{1}\left|f(x)-y_{1}(x)\right|^{2} d x \\
& =\int_{0}^{1}\left|f^{(m+1)}\left(\xi_{x}\right)\right|^{2}\left(\frac{x^{m+1}}{(m+1) !}\right)^{2} d x \\
& \leq \frac{\widehat{K}^{2}}{(m+1) !^{2}} \int_{0}^{1} x^{2 m+2} d x \\
& =\frac{\widehat{K}^{2}}{(m+1) !^{2}(2 m+3)} .
\end{aligned}
$$

Then by taking square roots, the proof is complete.

The previous theorem shows that the error vanishes as $m \rightarrow \infty$.
TABLE 3: Absolute errors by using BPFs for $N=4$, BPs for $M=3$, and HBBPFM for $N=4, M=3$ in Example 2.

\begin{tabular}{lccc}
\hline & & Method & \\
$t$ & BPFs & BPs & HBBPFM \\
& $N=4$ & $M=3$ & $N=4, M=3$ \\
\hline 0 & 0.134438 & 0.000939946 & $2.60043 \times 10^{-6}$ \\
0.1 & 0.0292675 & 0.000210236 & $6.00397 \times 10^{-7}$ \\
0.2 & 0.0869644 & 0.000396173 & $1.08124 \times 10^{-6}$ \\
0.3 & 0.103935 & 0.000126329 & $1.37399 \times 10^{-6}$ \\
0.4 & 0.0380311 & 0.000213179 & $7.99831 \times 10^{-7}$ \\
0.5 & 0.216077 & 0.00037144 & $4.28735 \times 10^{-6}$ \\
0.6 & 0.0426798 & 0.000246979 & $9.89894 \times 10^{-7}$ \\
0.7 & 0.148954 & 0.0000965353 & $1.78268 \times 10^{-6}$ \\
0.8 & 0.167943 & 0.000412916 & $2.26538 \times 10^{-6}$ \\
0.9 & 0.0661188 & 0.000254268 & $1.31873 \times 10^{-6}$ \\
\hline
\end{tabular}

TABLE 4: Absolute errors by using BPFs for $N=5$, BPs for $M=4$, and HBBPFs for $N=5, M=4$ in Example 2 .

\begin{tabular}{lccc}
\hline & & Method & \\
$t$ & BPFs & BPs & HBBPFM \\
& $N=5$ & $M=4$ & $N=5, M=4$ \\
\hline 0 & 0.106159 & 0.0000526416 & $1.44355 \times 10^{-8}$ \\
0.1 & 0.000988576 & 0.0000210365 & $1.12472 \times 10^{-9}$ \\
0.2 & 0.128144 & $8.80224 \times 10^{-6}$ & $1.65937 \times 10^{-8}$ \\
0.3 & 0.000312002 & 0.0000141662 & $1.06195 \times 10^{-9}$ \\
0.4 & 0.155374 & 0.0000171016 & $9.87722 \times 10^{-9}$ \\
0.5 & 0.00152224 & $7.80052 \times 10^{-7}$ & $2.99127 \times 10^{-9}$ \\
0.6 & 0.189012 & 0.0000166986 & $4.29645 \times 10^{-8}$ \\
0.7 & 0.00262215 & 0.0000156271 & $7.92177 \times 10^{-9}$ \\
0.8 & 0.230475 & $7.94076 \times 10^{-6}$ & $4.74483 \times 10^{-10}$ \\
0.9 & 0.00358729 & 0.0000229948 & $9.2012 \times 10^{-9}$ \\
\hline
\end{tabular}

Corollary 5. One can write $c^{T}\langle B, B\rangle \cong\langle f, B\rangle$, such that one defines $Q=\langle B, B\rangle$ that is a $(m+1) \times(m+1)$ matrix and is said dual matrix of $B$, and one can obtain

$$
\begin{aligned}
Q_{i+1, j+1} & =\int_{0}^{1} B_{i, m}(x) B_{j, m}(x) d x \\
& =\frac{\left(\begin{array}{c}
m \\
i
\end{array}\right)\left(\begin{array}{c}
m \\
j
\end{array}\right)}{(2 m+1)\left(\begin{array}{c}
2 m \\
i+j
\end{array}\right)}, \quad i, j=0,1, \ldots, m .
\end{aligned}
$$

Proof. We know

$$
f \cong s_{0}=\sum_{i=0}^{m} c_{i} B_{i, m}=c^{T} B ;
$$

therefore, the proof is complete.

Corollary 6. A function $f(t) \in L^{2}([0,1])$ may be expanded as follows:

$$
f(t)=\sum_{n=1}^{\infty} \sum_{m=0}^{\infty} p_{n, m} H_{n, m}(t) .
$$




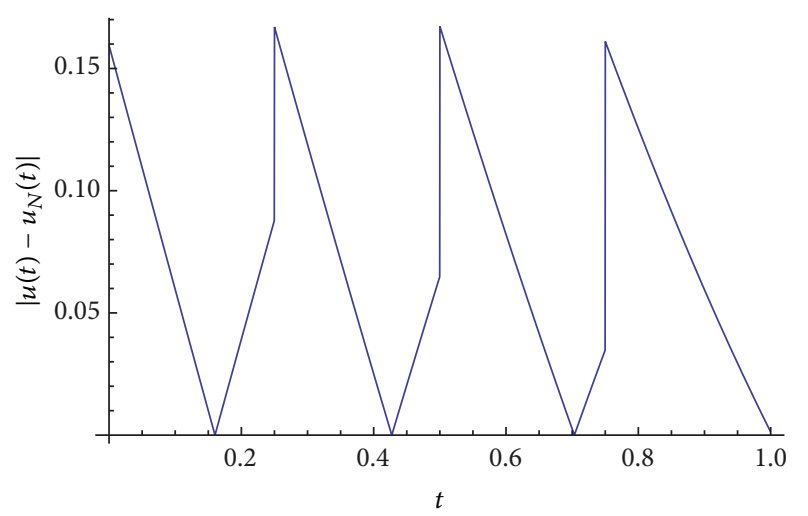

(a)

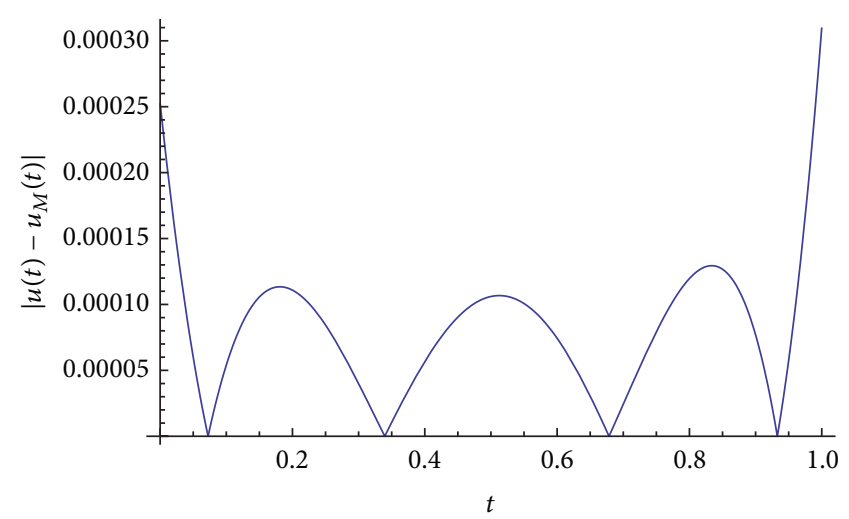

(b)

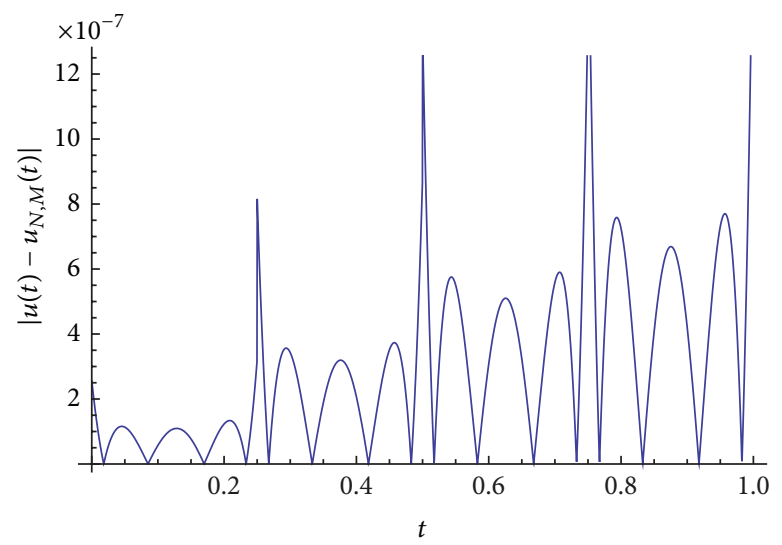

(c)

Figure 1: Plot of error functions by using BPFs for $N=4$ (a), BPs for $M=3$ (b), and HBBPFM for $N=4, M=3$ (c) in Example 1 .

If the infinite series in (16) is truncated, then we have

$$
f(t) \approx \sum_{n=1}^{N} \sum_{m=0}^{M} p_{n, m} H_{n, m}(t)=P^{T} H(t)
$$

where

$$
\begin{array}{r}
H(t)=\left[H_{1,0}(t), H_{1,1}(t), \ldots, H_{1, M}(t),\right. \\
\left.H_{2,0}(t), H_{2,1}(t), \ldots, H_{N, M}(t)\right]^{T}, \\
P=\left[p_{1,0}, p_{1,1}, \ldots, p_{1, M}, p_{2,0}, p_{2,1}, \ldots, p_{N, M}\right]^{T} .
\end{array}
$$

Therefore we can get

$$
P^{T}\langle H(t), H(t)\rangle=\langle f(t), H(t)\rangle .
$$

Then

$$
P=D^{-1}\langle f(t), H(t)\rangle,
$$

where

$$
\begin{aligned}
D & =\langle H(t), H(t)\rangle=\int_{0}^{1} H(t) H^{T}(t) d t \\
& =\left[\begin{array}{cccc}
D_{1} & 0 & \cdots & 0 \\
0 & D_{2} & \cdots & 0 \\
\vdots & \vdots & \ddots & \vdots \\
0 & 0 & \cdots & D_{N}
\end{array}\right],
\end{aligned}
$$

where by using (7), $D_{n}(n=1,2, \ldots, N)$ is defined as follows:

$$
\begin{aligned}
\left(D_{n}\right)_{i+1, j+1} & =\int_{(n-1) / N}^{n / N} B_{i, M}(N t-n+1) B_{j, M}(N t-n+1) d t \\
& =\frac{1}{N} \int_{0}^{1} B_{i, M}(t) B_{j, M}(t) d t \\
& =\frac{\left(\begin{array}{c}
M \\
i
\end{array}\right)\left(\begin{array}{c}
M \\
j
\end{array}\right)}{N(2 M+1)\left(\begin{array}{c}
2 M \\
i+j
\end{array}\right)}, \quad i, j=0,1, \ldots, M .
\end{aligned}
$$

We can also approximate the function $k(t, s) \in L^{2}([0,1] \times$ $[0,1])$ as follows:

$$
k(t, s) \approx H^{T}(t) K H(s),
$$




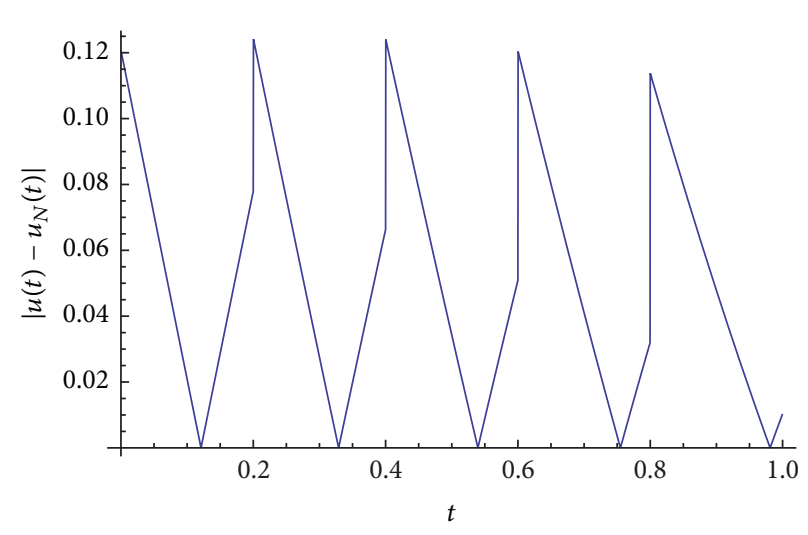

(a)

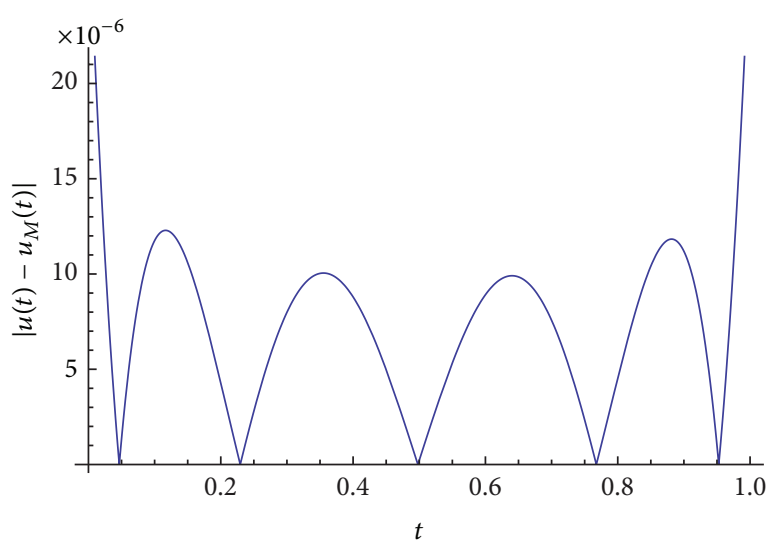

(b)

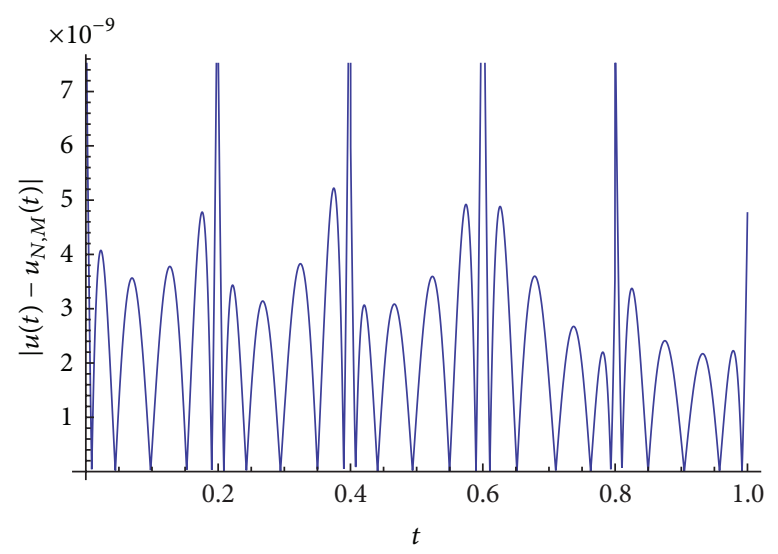

(c)

Figure 2: Plot of error functions by using BPFs for $N=5$ (a), BPs for $M=4$ (b), and HBBPFM for $N=5, M=4$ (c) in Example 1 .

where $K$ is an $N(M+1) \times N(M+1)$ matrix that we can obtain as follows:

$$
K=D^{-1}\langle H(t),\langle k(t, s), H(s)\rangle\rangle D^{-1}
$$

Theorem 7. Let the function $f:[0,1] \rightarrow R$ be $M+1$ times continuously differentiable; then we have

$$
\left\|f-P^{T} H\right\|_{L^{2}[0,1]} \leq \frac{\widetilde{K}}{N^{M+1}(M+1) ! \sqrt{2 M+3}}
$$

where $\widetilde{K}=\max _{t \in[0,1]}\left|f^{(M+1)}(t)\right|$.

Proof. By using Theorem 4 we get

$$
\begin{aligned}
\| f & -P^{T} H \|_{L^{2}[0,1]}^{2} \\
& =\int_{0}^{1}\left|f(t)-P^{T} H(t)\right|^{2} d x
\end{aligned}
$$

$$
\begin{aligned}
& =\sum_{n=1}^{N}\left(\int_{(n-1) / N}^{n / N} \mid f(t)\right. \\
& \left.\quad-\sum_{m=0}^{M} p_{n, m} B_{m, M}(N t-n+1) \mid\right) d t \\
& =\frac{1}{N} \sum_{n=1}^{N} \int_{0}^{1}\left|f\left(\frac{t+n-1}{N}\right)-\sum_{m=0}^{M} p_{n, m} B_{n, m}(t)\right|^{2} d t \\
& \leq \frac{1}{N^{2 M+3}} \sum_{n=1}^{N} \int_{0}^{1}\left|f^{(M+1)}\left(\xi_{n}\right)\right|^{2} \frac{t^{2 M+2}}{(M+1) !^{2}} d t \\
& \leq \frac{1}{N^{2 M+3}} \sum_{n=1}^{N} \frac{\widehat{K}_{n}^{2}}{(M+1) !^{2}(2 M+3)} \\
& \leq \frac{\widetilde{K}^{2}}{N^{2 M+2}(M+1) !^{2}(2 M+3)},
\end{aligned}
$$

where $\xi_{n} \in((n-1) / N, n / N)$ and $\widehat{K}_{n}=$ $\max _{t \in[(n-1) / N, n / N]}\left|f^{(M+1)}(t)\right|$. Therefore by taking square roots, the proof is complete. 


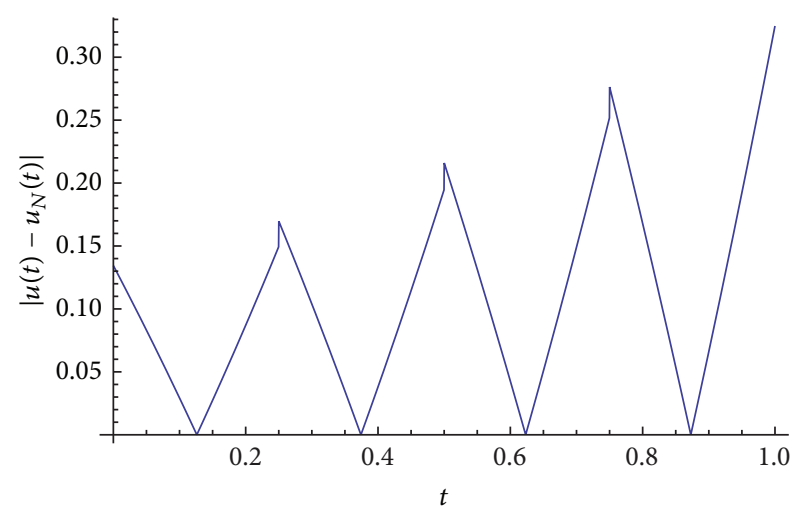

(a)

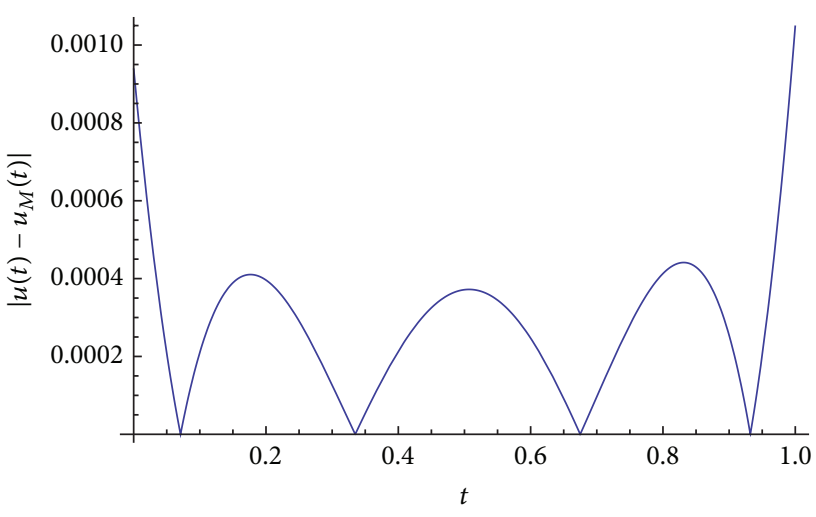

(b)

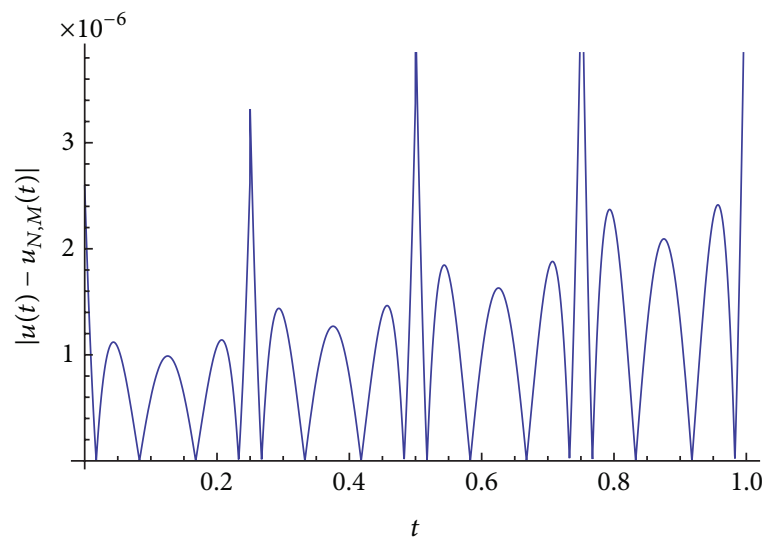

(c)

FIgURE 3: Plot of error functions by using BPFs for $N=4$ (a), BPs for $M=3$ (b), and HBBPFM for $N=4, M=3$ (c) in Example 2 .

The above theorem shows that the approximation error vanishes as $M, N \rightarrow \infty$.

\section{HBBPFs for the Second Kind Integral Equations and Error Analysis}

In this section, we are dealing with the following Fredholm equations of the second kind:

$$
u(t)=\int_{0}^{1} k(t, s) u(s) d s+f(t)
$$

where $u, f \in L^{2}([0,1]), k \in L^{2}([0,1] \times[0,1])$, and $u(t)$ is an unknown function.

Let us approximate $u, f$, and $k$ by (18) and (25) as follows:

$$
\begin{gathered}
u(t) \approx U^{T} H(t), \quad f(t) \approx F^{T} H(t), \\
k(t, s) \approx H^{T}(t) K H(t) .
\end{gathered}
$$

By substituting (29) in (28) we obtain

$$
\begin{aligned}
H^{T}(t) U & =\int_{0}^{1} H^{T}(t) K H(s) H^{T}(s) U d s+H^{T}(t) F \\
& =H^{T}(t) K\left(\int_{0}^{1} H(s) H^{T}(s) d s\right) U+H^{T}(t) F \\
& =H^{T}(t) K D U+H^{T}(t) F=H^{T}(t)(K D U+F) .
\end{aligned}
$$

Therefore we have the following linear system:

$$
(I-K D) U=F,
$$

that by solving this linear system we can obtain the vector $U$.

Theorem 8. Suppose that $u(t)$ is exact solution of (28) and $u_{N, M}(t)$ is approximate solution by HBBPFs for $u(t)$ and $E_{N, M}(t)$ is perturbation function that depends only on $u_{N, M}(t)$ (i.e., $u_{N, M}(t)=\int_{0}^{1} k(t, s) u_{N, M}(s) d s+f(t)+$ $\left.E_{N, M}(t)\right)$. Let $R=\max _{0 \leq t, s \leq 1}|k(s, t)|<\infty$. Then $E_{N, M}(t) \rightarrow 0$ as $M, N \rightarrow \infty$. 


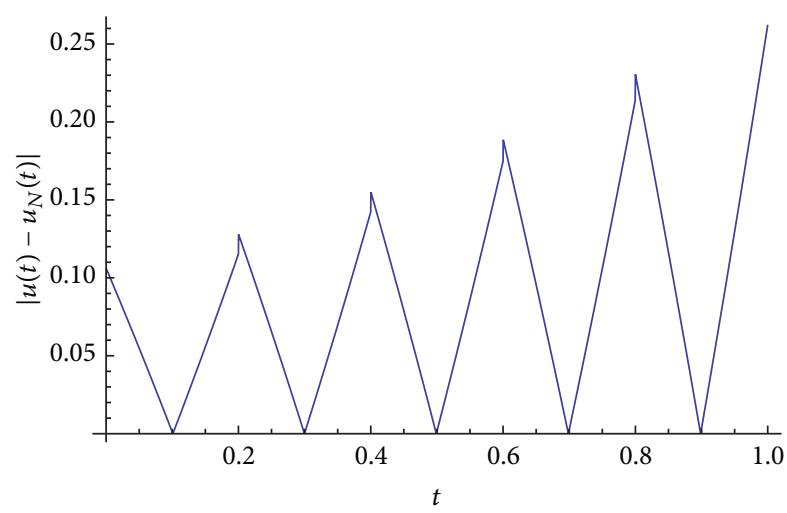

(a)

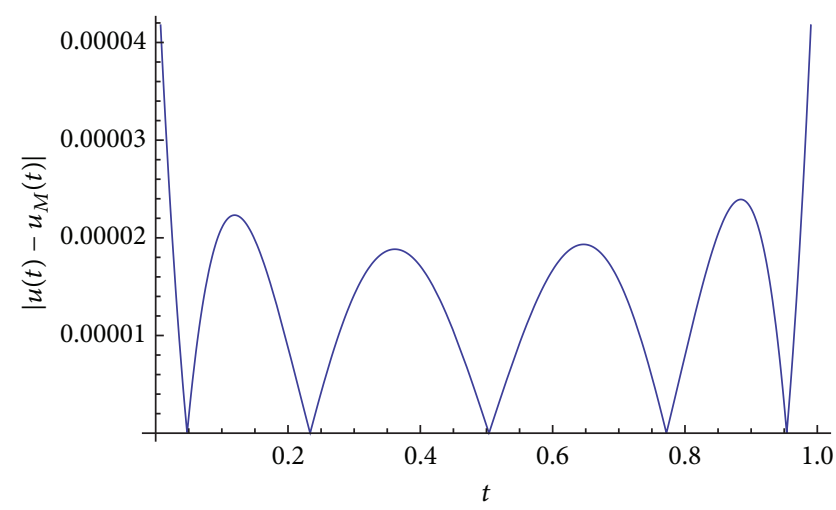

(b)

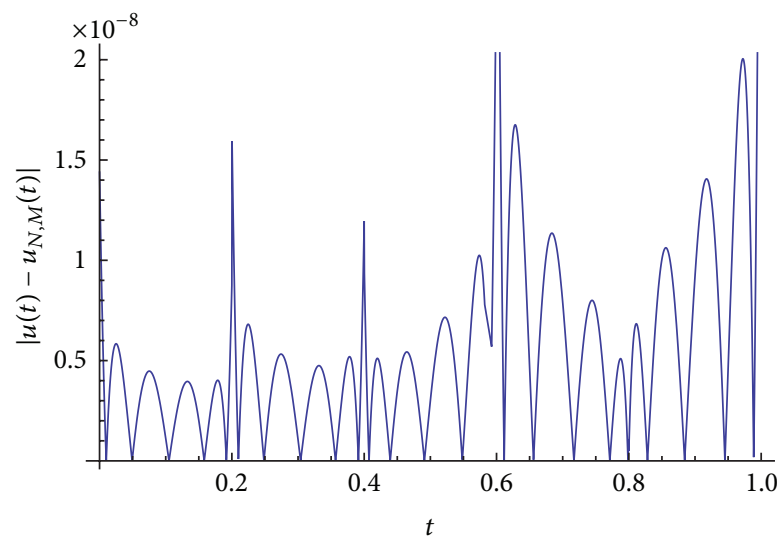

(c)

FIgURE 4: Plot of error functions by using BPFs for $N=5$ (a), BPs for $M=4$ (b), and HBBPFM for $N=5, M=4$ (c) in Example 2.

Proof. Suppose $e_{N, M}(t)=u(t)-u_{N, M}(t)$ is the error function of approximate solution $u_{N, M}(t)$ to the exact solution $u(t)$. Therefore we get

$$
e_{N, M}(t)=\int_{0}^{1} k(t, s) e_{N, M}(s) d s-E_{N, M}(t)
$$

By taking absolute value and using Holder inequality we get

$$
\begin{aligned}
\left|E_{N, M}(t)\right| \leq & \int_{0}^{1}|k(t, s)|\left|e_{N, M}(s)\right| d s+\left|e_{N, M}(t)\right| \\
\leq & \left(\int_{0}^{1}|k(t, s)|^{2} d s\right)^{1 / 2}\left(\int_{0}^{1}\left|e_{N, M}(t)\right|^{2} d s\right)^{1 / 2} \\
& +\left|e_{N, M}(t)\right| \\
\leq & R\left\|e_{N, M}(t)\right\|_{L^{2}[0,1]}+\left|e_{N, M}(t)\right| .
\end{aligned}
$$

Now, by taking norm $L^{2}([0,1])$ we obtain

$$
\left\|E_{N, M}(t)\right\|_{L^{2}[0,1]} \leq(R+1)\left\|e_{N, M}(t)\right\|_{L^{2}[0,1]} .
$$

Finally, from Theorem 7 we can write

$$
\left\|E_{N, M}(t)\right\|_{L^{2}[0,1]} \leq \frac{(R+1) \bar{K}}{N^{M+1}(M+1) ! \sqrt{2 M+3}},
$$

where $\bar{K}=\max _{t \in[0,1]}\left|u^{(M+1)}(t)\right|$. $\infty$.

Therefore, we can show that $E_{N, M}(t) \rightarrow 0$ as $M, N \rightarrow$

\section{Numerical Examples}

In this section we discuss the implementation of the new method and investigate its accuracy by applying it to different examples. In the following examples, we suppose that $u_{N}(t)$, $u_{M}(t)$, and $u_{M, N}(t)$ are approximate solutions by BPFs, BPs, and HBBPFM for the exact solution $u(t)$, respectively.

Example 1. Consider the following integral equation:

$$
\begin{aligned}
u(t)= & \int_{0}^{1}(t+s) u(s) d s+\sin (t) \\
& \quad-t+(t+1) \cos (1)-\sin (1) .
\end{aligned}
$$

We know that the exact solution is $u(t)=\sin (t)$. The obtained results of BPFs, BPs, and HBBPFs are reported in Tables 1 and 2 and are plotted in Figures 1 and 2. We compare the obtained results and observe that HBBPFM is very effective and accuracy of approximate solutions in this method is more than methods of BPFs and BPs. 
Example 2. Consider the following integral equation:

$$
u(t)=\int_{0}^{1} t s u(s) d s+e^{t}-t
$$

with exact solution $u(t)=e^{t}$. We obtain the computational by BPFs, BPs, and HBBPFM with $N=4, M=3$, and $N=5$, $M=4$; then we compare them together. The results are reported in Tables 3 and 4 and are plotted in Figures 3 and 4. Similar to the previous example, we see that the method HBBPFM is very effective and accuracy of solution in this method is more than methods of BPFs and BPs.

\section{Conclusion}

In this paper, $\mathrm{HBBPF}$ are used to solve second kind integral equations we call this method with HBBPFM. This method converts second kind integral equations to systems of linear equations whose answers are coefficient of HBBPFs expansion of the solution of second kind integral equations. Also, by using several lemmas and theorems, we have discussed convergence analysis of the proposed method. Numerical examples show the efficiency and accuracy of the method. Moreover we see that accuracy of solutions in HBBPFM is more satisfactory than the methods of BPFs and BPs.

\section{Conflict of Interests}

The authors declare that there is no conflict of interests in this paper.

\section{References}

[1] E. Babolian and Z. Masouri, "Direct method to solve Volterra integral equation of the first kind using operational matrix with block-pulse functions," Journal of Computational and Applied Mathematics, vol. 220, no. 1-2, pp. 51-57, 2008.

[2] K. Maleknejad and B. Rahimi, "Modification of block pulse functions and their application to solve numerically Volterra integral equation of the first kind," Communications in Nonlinear Science and Numerical Simulation, vol. 16, no. 6, pp. 24692477, 2011.

[3] F. Mirzaee and S. Piroozfar, "Numerical solution of the linear two-dimensional Fredholm integral equations of the second kind via two-dimensional triangular orthogonal functions," Journal of King Saud University - Science, vol. 22, no. 4, pp. 185193, 2010.

[4] Y. Ordokhani, "Solution of nonlinear Volterra-FredholmHammerstein integral equations via rationalized Haar functions," Applied Mathematics and Computation, vol. 180, no. 2, pp. 436-443, 2006.

[5] H. R. Marzban, H. R. Tabrizidooz, and M. Razzaghi, "A composite collocation method for the nonlinear mixed VolterraFredholm-Hammerstein integral equations," Communications in Nonlinear Science and Numerical Simulation, vol. 16, no. 3, pp. 1186-1194, 2011.

[6] M. Tavassoli Kajani and A. Hadi Vencheh, "Solving second kind integral equations with hybrid Chebyshev and block-pulse functions," Applied Mathematics and Computation, vol. 163, no. 1, pp. 71-77, 2005.
[7] X. T. Wang and Y. M. Li, "Numerical solutions of integrodifferential systems by hybrid of general block-pulse functions and the second Chebyshev polynomials," Applied Mathematics and Computation, vol. 209, no. 2, pp. 266-272, 2009.

[8] K. Maleknejad and Y. Mahmoudi, "Numerical solution of linear Fredholm integral equation by using hybrid Taylor and blockpulse functions," Applied Mathematics and Computation, vol. 149, no. 3, pp. 799-806, 2004.

[9] B. Asady, M. Tavassoli Kajani, A. Hadi Vencheh, and A. Heydari, "Solving second kind integral equations with hybrid Fourier and block-pulse functions," Applied Mathematics and Computation, vol. 160, no. 2, pp. 517-522, 2005.

[10] G. Prasada Rao, Piecewise Constant Orthogonal Functions and their Application to Systems and Control, Springer, Berlin, Germany, 1983.

[11] B. M. Mohan and K. B. Datta, Orthogonal Functions in Systems and Control, 1995.

[12] E. H. Doha, A. H. Bhrawy, and M. A. Saker, "Integrals of Bernstein polynomials: an application for the solution of high even-order differential equations," Applied Mathematics Letters, vol. 24, no. 4, pp. 559-565, 2011.

[13] B. N. Mandal and S. Bhattacharya, "Numerical solution of some classes of integral equations using Bernstein polynomials," Applied Mathematics and Computation, vol. 190, no. 2, pp. 17071716, 2007.

[14] S. A. Yousefi and M. Behroozifar, "Operational matrices of Bernstein polynomials and their applications," International Journal of Systems Science, vol. 41, no. 6, pp. 709-716, 2010.

[15] M. Alipour and D. Rostamy, "Solving nonlinear fractional differential equations by Bernstein polynomials operational matrices," The Journal of Mathematics and Computer Science, vol. 5, no. 3, pp. 185-196, 2012.

[16] D. Rostamy, M. Alipour, H. Jafari, and D. Baleanu, "Solving multi-term orders fractional differential equations by operational matrices of BPs with convergence analysis," Romanian Reports in Physics, vol. 65, no. 2, pp. 334-349, 2013.

[17] D. Baleanu, M. Alipour, and H. Jafari, “The Bernstein operational matrices for solving the fractional quadratic Riccati differential equations with the Riemann-Liouville derivative," Abstract and Applied Analysis, vol. 2013, Article ID 461970, 7 pages, 2013.

[18] M. Alipour and D. Baleanu, "Approximate analytical solution for nonlinear system of fractional differential equations by BPs operational matrices," Advances in Mathematical Physics, vol. 2013, Article ID 954015, 9 pages, 2013.

[19] M. Alipour, D. Rostamy, and D. Baleanu, "Solving multidimensional fractional optimal control problems with inequality constraint by Bernstein polynomials operational matrices," Journal of Vibration and Control, vol. 19, no. 16, pp. 2523-2540, 2013.

[20] M. Alipour and D. Rostamy, "BPs operational matrices for solving time varying fractional optimal control problems," The Journal of Mathematics and Computer Science, vol. 6, pp. 292304, 2013.

[21] K. Atkinson and W. Han, Theoretical Numerical Analysis: A Functional Analysis Framework, Springer, 2nd edition, 2000. 


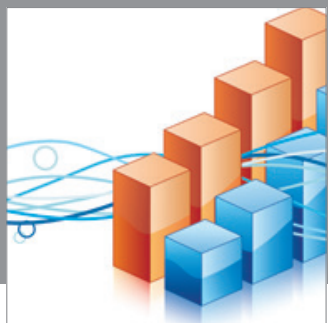

Advances in

Operations Research

mansans

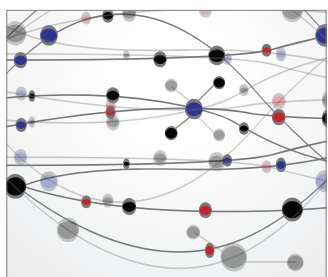

The Scientific World Journal
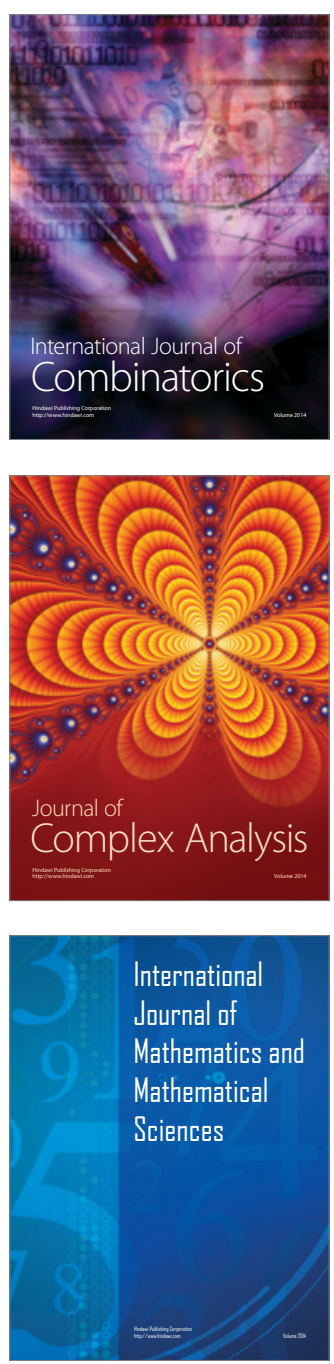
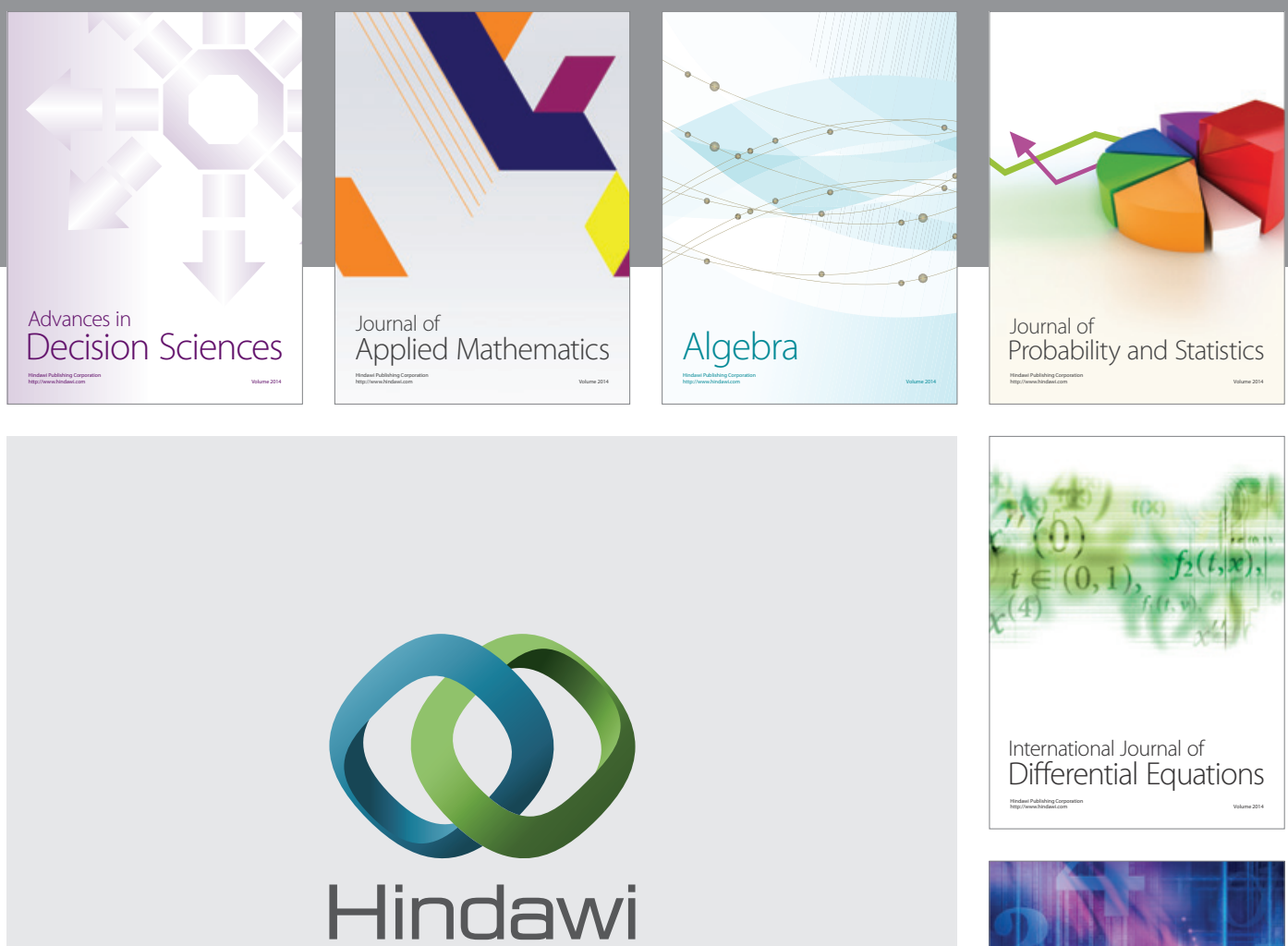

Submit your manuscripts at http://www.hindawi.com
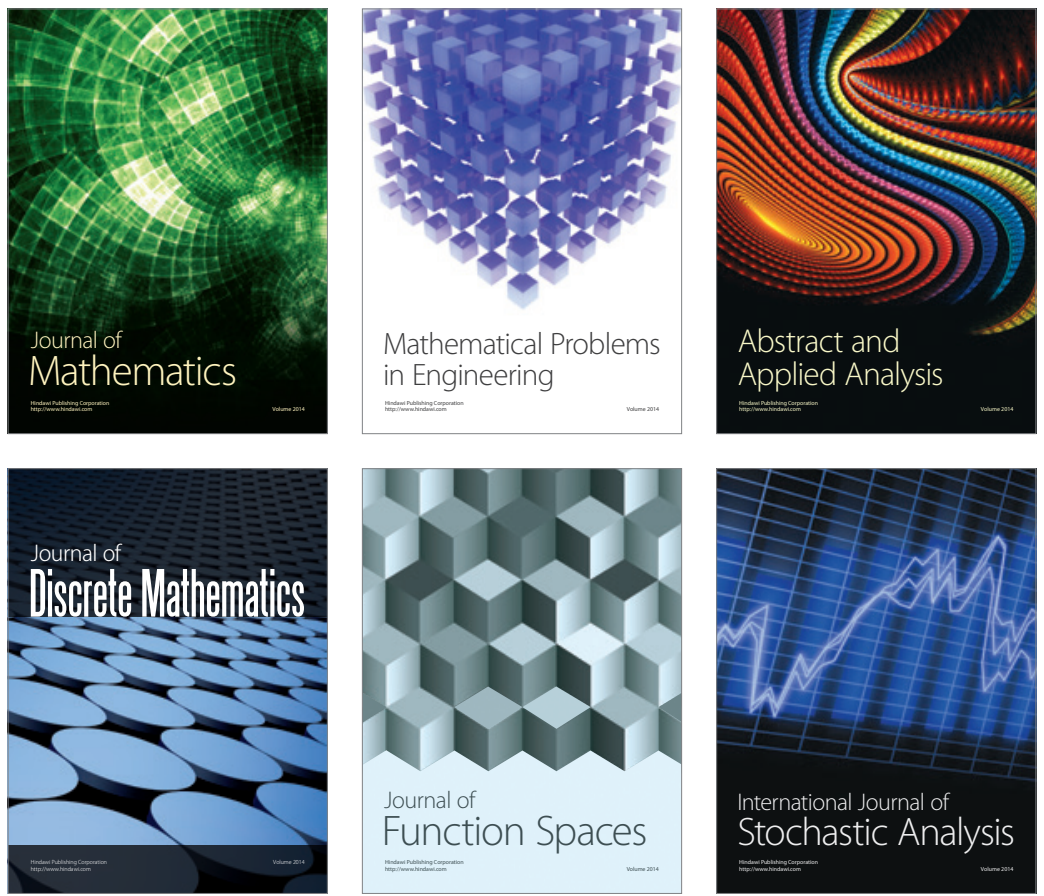

Journal of

Function Spaces

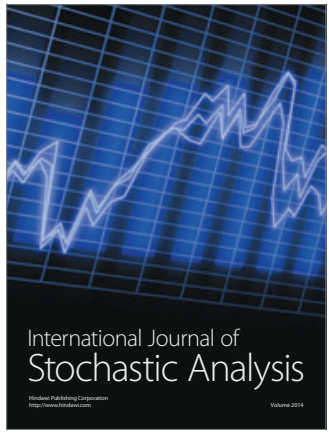

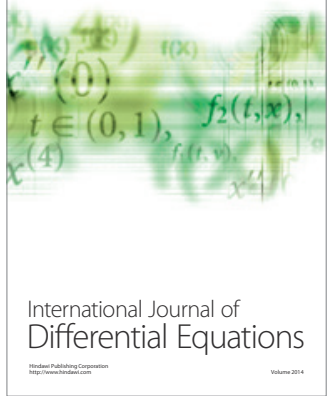
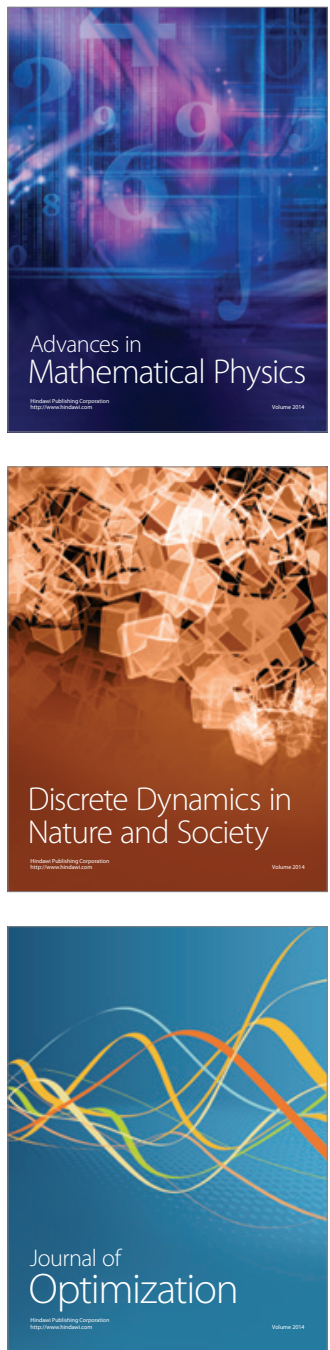